\title{
Valoración de acciones en los países que integran la Alianza del Pacífico por el modelo de descuento de dividendos*
}

\author{
Stock valuation in Pacific Alliance countries using the dividend discount model \\ Fray Alonso Betancur-Duque (iD) \\ Estudiante de Ingeniería Financiera, Instituto Tecnológico Metropolitano, \\ Medellín-Colombia, fraybetancur64534@correo.itm.edu.co \\ Edwin Andrés Jiménez-Echeverri \\ Magíster en Administración Financiera, Instituto Tecnológico Metropolitano, \\ Medellín-Colombia, edwinjimenez@itm.edu.co
}

\begin{abstract}
Cómo citar / How to cite
Betancur-Duque, F. A. \& Jiménez-Echeverri, E. A. (2019). Valoración de acciones en los países que integran la Alianza del Pacífico por el modelo de descuento de dividendos. Revista CEA, 5(10), 99-116. https://doi.org/10.22430/24223182.1450
\end{abstract}

Recibido: 31 de enero de 2019

Aceptado: 20 de junio de 2019

\section{Resumen}

El objetivo de esta investigación es analizar las oportunidades de inversión en los países pertenecientes a la Alianza del Pacífico, usando el modelo de descuento de dividendos con empresas que operan en estos países, que poseen acciones bursátiles. Para esta investigación se usó el software Economática, mediante el cual se recopilaron los datos correspondientes al precio de cierre para las acciones negociadas en abril de 2018, dividendos por acción, utilidad por acción, coeficiente beta y Rentabilidad del patrimonio; posteriormente, se realizó la valoración de acciones y la comparación de los resultados con los índices MSCl - Morgan Stanley Capital International, para la Alianza del Pacífico. En los resultados obtenidos, se observa una sobrevaloración generalizada de las acciones estudiadas. Se destaca el caso de México, con una sobrevaloración por encima del promedio, una alta volatilidad y la mayor rentabilidad promedio sobre el patrimonio. También se observó una considerable relación entre la rentabilidad del patrimonio y la tasa de retención de utilidades en las acciones estudiadas. Los índices de sobrevaloración generan la hipótesis de dividendos ineficientes para representar una expectativa clara sobre el valor de la compañía, por lo tanto, en la presente investigación, el modelo de descuento de dividendos no estaría facultado para mostrar de manera eficiente el valor de todas las compañías; por otro lado, también es posible que la ineficiencia resulte de la metodología usada para determinar la tasa de descuento, generando una tasa descuento mayor a la que implícitamente acepta el mercado. A pesar de lo anterior, la valoración por el modelo de

\footnotetext{
* Este artículo se deriva del proyecto titulado "Valoración de acciones en los países que integran la Alianza del Pacífico por el modelo de descuento de dividendos" y ha sido financiado con recursos propios.
} 
descuento de dividendos sirve como referente de las oportunidades de inversión en las empresas pertenecientes a la Alianza del Pacífico.

Palabras clave: modelo de descuento de dividendos, análisis financiero bursátil, métodos de valoración, Alianza del Pacífico.

\begin{abstract}
The objective of this study is to analyze investment opportunities in publicly traded firms in Pacific Alliance countries using the dividend discount model. The software Economática was employed to collect multiple data: closing price of stocks traded in April 2018, dividends per share, earnings per share, beta coefficient, and Return on Equity (ROE). Subsequently, the stocks were valued, and the outcome was compared with the $\mathrm{MSCl}$ (Morgan Stanley Capital International) indexes for the Pacific Alliance. The results show a generalized overvaluation of the stocks under study. Mexico stands out with an above-average overvaluation, high volatility, and greater average ROE. A significant relationship was also observed between the ROE and the plowback ratio of such stocks. The overvaluation indexes suggest the hypothesis that dividends cannot efficiently represent a clear expectation of the value of a company. However, such inefficiency could be caused by the methodology adopted to determine the discount rate, which generates a discount rate higher than the market implicitly accepts. Regardless, the valuation by the dividend discount model can serve as a reference point for investment opportunities in companies in the Pacific Alliance.
\end{abstract}

Keywords: Dividend discount model, financial stock analysis, valuation methods, Pacific Alliance.

\title{
1. INTRODUCCIÓN
}

La Alianza del Pacífico fue establecida en 2011 como mecanismo para la integración política, económica y de cooperación entre Chile, Colombia, México y Perú. Dadas las mayores posibilidades de adquisiciones y fusiones entre las empresas de los países que componen la Alianza, se hace fundamental para los interesados conocer el valor intrínseco de las acciones que cotizan en bolsa pues a partir de estas negociaciones es que se logra un punto de referencia transaccional. Adicionalmente, de debe considerar que una de las intenciones en la Alianza es «avanzar hacia la libre circulación de capitales y la promoción de las inversiones entre las partes» (Acuerdo Marco de la Alianza del Pacífico, 2012, p. 4). Se hace relevante, entonces, trabajar en metodologías de valoración que sirvan de referente para una circulación de capitales que promueva negociaciones en condiciones justas.

Adicionalmente, debido a las expectativas de libre circulación de capitales asociada a los mecanismos para la integración económica entre países y ante la necesidad de reducción de la incertidumbre respecto al valor de las compañías en los cuales se abre el espectro de inversión, se hace evidente la necesidad de acoger un modelo de valoración de empresas que permita una velocidad de circulación eficiente de los capitales en el mercado de valores, evitando largos y dispendiosos procesos de valoración debido a la posibilidad de apalancarse en la mayor auditoría que ya ejercen los organismos de control para las empresas que cotizan en este mercado; por lo tanto, debemos considerar que la valoración fundamental también catalogada por algunos autores como valoración relativa, «es un método de análisis cuyo fin no es el cálculo de un valor absoluto para una compañía, sino una 
comparación para determinar si la compañía en cuestión está cara o barata en términos relativos al mercado» (Hernández, 2007, p. 165).

Sobre la base de las consideraciones anteriores, se tiene en cuenta el estudio realizado por Damodaran (2007), quien encontró tres tendencias clave en la valoración de empresas, en las décadas recientes. En primer lugar, el uso del modelo de descuento de dividendos; segundo, las conexiones entre las finanzas corporativas y la valoración se han vuelto más claras, ya que el valor está vinculado al comportamiento de la empresa; y, en tercer lugar, la conclusión de que todos los modelos conducen a equivalentes valores. Dando continuidad a la primera tendencia mencionada, se puede inferir que uno de los mecanismos de valoración que ha demostrado mayor eficiencia al inyectarle dinamismos a los procesos de circulación del mercado de valores norteamericano es el modelo de descuento de dividendos (DDM, por sus siglas en inglés: Dividend Discount Model), el cual ha sido usado por bancas de inversión, como JP Morgan, en la valuación y el proceso de selección de acciones e incluso, algunas de ellas como el First Boston, publicaron sus cálculos periódicamente basados en este modelo (Van Horne, 1997, p. 29).

Debido al limitado tamaño del mercado de valores en Latinoamérica, la experiencia con la toma de decisiones de inversión basados en el uso del DDM es menor, sin embargo, el punto de partida de la presente investigación es la siguiente pregunta: ¿Es posible estudiar las oportunidades de inversión en los países pertenecientes a la Alianza del Pacífico, usando el modelo de descuento de dividendos? Lo anterior nos lleva a plantear como objetivo general de la investigación, la elaboración de un análisis sobre las oportunidades de inversión en los países pertenecientes a la Alianza del Pacífico, usando el modelo de descuento de dividendos.

Por todo lo expuesto, el análisis posee un componente de validación del modelo teórico que resulta relevante en los resultados presentados. Adicionalmente, para identificar las oportunidades de mercado en la Alianza es importante conocer el comportamiento del valor de las compañías que la integran.

El presente artículo consta de cuatro partes principales: en la primera, se presenta el soporte conceptual de los modelos de descuento de dividendos y Modelo de Fijación de precios de activos de capital o CAPM (Capital Asset Pricing Model); la segunda, contempla la metodología aplicada, en la que los datos base para los cálculos, se recopilaron del software Economática; en la tercera, se presentan de forma descriptiva, los resultados de la investigación y se describen las conclusiones y posibles trabajos futuros.

\section{MARCO TEÓRICO}

Entre las definiciones con respecto al concepto de eficiencia de los mercados, es posible encontrar que:

Un mercado 'eficiente' es un mercado donde hay un gran número de agentes racionales, que compiten activamente en búsqueda de la máxima ganancia, cada uno tratando de predecir el precio de mercado futuro de tal o cual título, y donde la información corriente está a disposición de manera casi libre para todos los participantes. Por tanto, en un mercado eficiente, en cualquier momento, el precio actual de un título sería una buena estimación de su valor intrínseco (Fama, 1965, p 3-4). 
De acuerdo con esto, si el mercado es eficiente debe recoger los fundamentales de la compañía en el largo plazo y, por ende, el valor de mercado se debe acercar al valor intrínseco de la acción. Algunas investigaciones hacen alusión a la importancia del uso de metodologías de valoración para la compra de organizaciones, en especial si se tiene en cuenta, que el precio de la acción no siempre corresponde a su valor contable, tal como lo indica Hernández (2012):

Es evidente que en la ciencia contable se adaptan metodologías a registros sistémicos a partir de directrices jurídicas, las cuales no siempre se ajustan plenamente a la realidad financiera. Esta conclusión, se hace evidente cuando se coteja el valor de una acción en libros y su valor en el mercado de valores (p. 47).

Es importante recalcar, que no se debe confundir valor y precio. El precio se determina en el mercado de acuerdo con las leyes de oferta y demanda, mientras que, respecto al valor, existen numerosos métodos de valoración, entre ellos «métodos basados en el balance de la empresa; métodos basados en la cuenta de resultados; métodos mixtos, y métodos basados en el descuento de flujos de fondos» (Fernández, 2008, p. 4). Cabe agregar que «Los métodos conceptualmente "correctos" para valorar empresas con expectativas de continuidad, son los basados en el descuento de flujos de fondos, los cuales consideran a la empresa como un ente generador de flujos de fondos» (Fernández, 2008, p. 3).

Uno de los métodos más conocidos es el Modelo de Gordon y Shapiro, que determina el valor teórico o intrínseco de una acción, en función del valor de sus dividendos futuros y de la tasa de crecimiento de los mismos. En vista de que el modelo de descuento de dividendos (DDM, por sus siglas en inglés: Dividend Discount Model) es un modelo ampliamente usado, se aplicó en la presente investigación. Entre las primeras versiones del DDM es posible ubicar a Myron J. Gordon, quien en 1962 la publicó en The Investment, Financig and the Valuation of the Corporation (Sección 3.3.2). Posteriormente, se desarrollaron otras versiones. Sin embargo, aún causan descontentos algunos supuestos del modelo, como la capacidad de la compañía para producir utilidades y las políticas de distribución de estas, ya que una tasa diferente de retención de utilidades podría dar como resultado un valor intrínseco diferente.

Los modelos de descuento de dividendos (DDM) en su formulación más básica aplican la siguiente fórmula:

$$
V o=\sum_{t=1}^{\infty} \frac{D t}{(1+k e)^{\wedge} t}
$$

Donde $D t$ son los dividendos pagados en el momento $t$ y ke es la tasa de rentabilidad exigida a las acciones, ajustada a su nivel de riesgo.

Posterior, al uso de la fórmula (1), Gordon y Shapiro (1956) desarrollaron una simplificación del modelo, asumiendo una tasa de crecimiento futura constante de los dividendos, esto debido a «la necesidad de contrarrestar el efecto negativo que la inflación anual produce en los dividendos y la reinversión de las utilidades por acción no distribuida en forma de dividendos, que se traduciría en unos mayores resultados para años sucesivos» (Jaramillo, 2009, p. 238). La formulación de este modelo simplificado puede expresarse así: 


$$
V o=\frac{D 1}{k e-g p} ; k e>g p(2)
$$

Donde gp es la tasa de crecimiento constante de los dividendos y se supone ke-gp>0.

Para determinar el ke existen diferentes métodos, entre los que podemos encontrar «modelo risk premium, el modelo de descuento de flujos de caja y los modelos de determinación del precio de los activos de capital Capital Asset Pricing Model (CAPM), el arbritrage Price theory (APT) y el three factor model (TFM)» (Milla, 2011, p. 34).

Entre los modelos para determinar el ke con mayor acogida y relevancia, se encuentra el modelo CAPM. Una encuesta llevada a cabo por la Asociación de Profesionales Financieros (AFP) a 300 organizaciones encontró que:

Una abrumadora mayoría de las organizaciones (87 por ciento) usan el modelo de fijación de precios de activos de capital (CAPM) al estimar su costo de capital. CAPM es el método preferido para estimar el costo del capital, independientemente del tamaño de la organización y el tipo de propiedad (Association for Financial Professionals, 2011, p. 9).

Por otra parte, el modelo CAPM «se basa en la hipótesis de eficiencia del mercado, asumiendo que la información relevante está totalmente disponible para todos los inversores y se refleja rápidamente en el precio de las acciones» (Vila, 2004, p. 32), por lo tanto:

El costo de capital de una empresa para una inversión o proyecto es el rendimiento esperado que sus inversionistas podrían ganar sobre otros valores, con el mismo riesgo y vencimiento. Debido a que el riesgo que determina los rendimientos esperados es sistémico, mismo que es medido por la beta, el costo de capital para una inversión es el rendimiento esperado disponible sobre valores con la misma beta (Berk \& Demarzo, 2008, p. 311).

Finalmente, esto no lleva a indicar que la ecuación que muestra la relación lineal entre el riesgo y el rendimiento es la siguiente:

$$
C A P M=R f+B_{1}\left(R_{m}-R_{F}\right)+\text { Riesgo país (3) }
$$

Donde:

$\mathrm{Rf}=$ Rentabilidad libre de riesgo

$B_{1}=$ Coeficiente de riesgo sistemático

$R_{m}=$ Rendimiento esperado de la cartera de mercado

\section{Estado del arte}

Anand (2002) encuestó a 81 directores de finanzas (CFO) del BT-500 de India para conocer sus prácticas financieras y encontró que el CAPM es el método más popular con 54,32\% de estimaciones del costo del capital social. El segundo y el tercer método más populares son: modelo de descuento de dividendos de Gordon (52.1 \%) y rendimiento de las ganancias (34.2 \%) respectivamente. 
Por su parte, Nasseh \& Strauss (2004) y Foerster \& Sapp (2005) examinaron la relación entre los precios de las acciones y el modelo de descuento de dividendos y encontraron una relación estable que respaldaba el modelo durante la mayor parte del período de muestra, para empresas que tienen una larga historia de pago de dividendos.

Adicionalmente, Jiang \& Lee (2005) y Charumathi \& Suraj (2014) realizaron estudios para establecer la fiabilidad del DDM, concluyeron que el modelo convencional de descuento de dividendos no puede explicar los precios volátiles de las acciones, ya que pueden estar relacionados con problemas tales como la medida de dividendos y factores de descuento o racionalidad de los inversores. De igual forma, Tareq (2012) realiza una investigación para comparar el modelo de Ingresos Residuales (RIM) y el modelo de descuento de dividendos (DDM), concluyendo que ambos modelos proporcionan las mismas estimaciones de valores, cuando se puede pronosticar el valor del terminal.

También, Göbel (2015) e Ivanovski, Ivanovska \& Narasanov (2015) realizaron valoraciones con el modelo de descuento de dividendos y encontraron que los precios medios de mercado y los precios medios de las acciones son significativamente más altos que sus valores intrínsecos. Por otra parte, Sim \& H. Wright (2017), utilizando el modelo de descuento de dividendos, adicionan el método de simulación de Monte Carlo para las acciones que conforman el promedio industrial Dow Jones, proponiendo de esta forma un uso alternativo del modelo de descuento de dividendos, para permitir que un inversor evalúe los riesgos asociados con una acción en particular, en función de su historial de dividendos.

Por otra parte, los estudios sobre el comportamiento de las acciones en bosas de valores con una característica común definida por el investigador, con el objetivo de analizar las oportunidades de inversión, es compartido por múltiples investigaciones, entre estas es posible resaltar el trabajo realizado por Quiroga-Juárez \& Villalobos-Escobedo (2015), en el cual las principales bolsas financieras en el mundo fueron seleccionadas como objeto de investigación y la metodología usada para el análisis de oportunidades de inversión fue la del análisis multivariado (análisis de componentes principales PCA), con lo cual además se demostró que este tipo de análisis es una poderosa herramienta, particularmente para el análisis técnico; aunque el objetivo y el objeto de estudio comparten características similares con la presente investigación, la valoración de acciones por medio del DDM, se considera una metodología para el análisis fundamental en lugar de las metodología de análisis técnico con la cual se produce el mayor número de investigaciones recientes asociadas al mercado bursátil.

\section{METODOLOGÍA}

La presente investigación inició con un estudio descriptivo sobre las oportunidades de inversión en los países pertenecientes a la Alianza del Pacífico; sin embargo, sus resultados llevan a la hipótesis sobre dividendos ineficientes para representar la expectativa sobre el valor de la compañía, o de una posible ineficiencia en la metodología usada para determinar la tasa de descuento, que podían encadenarse con estudios explicativos. Asimismo, el uso de un método de investigación deductivo que parte del uso del modelo de descuento de dividendos, busca analizar resultados concretos para las empresas que operan en los países asociados a la Alianza. 
Al mismo tiempo, la fuente de información es secundaria y se encuentra consignada en el software Economática, del cual se recopilaron los datos correspondientes al cierre (acciones negociadas), dividendos por acción, utilidad por acción, coeficiente beta de cada acción y roe inicial, para los países de la Alianza del Pacífico; durante el mes de abril de 2018 esta base de datos se exportó al software Excel.

Del conjunto de datos se seleccionaron las acciones que reportaron todos y cada uno de los ítems mencionados anteriormente. Filtrada esta matriz de datos, se procedió a calcular la tasa de pago de dividendos y, como complemento de esta, la tasa de retención de utilidades y la tasa de crecimiento de dividendos (gp), para luego, calcular el valor intrínseco de la acción, con el objeto de realizar su posterior análisis e interpretación; en síntesis, se calcularon todos los conceptos de los que trata el modelo de descuento de dividendos, así:

Tasa de retención, los dividendos se pueden expresar como un porcentaje de utilidad neta reportada, el porcentaje $b$ que se retiene.

Tasa de pago, el restante $1-b$, es la tasa de pago.

Tasa de crecimiento de los dividendos, el modelo Gordon de descuento de dividendos (DDMCG), asume que los dividendos crecen a la tasa gp, y 1- $b$ es constante, entonces las utilidades y el valor intrínseco, también están creciendo a la tasa gp.

Matemáticamente, se tiene:

$$
g p=(b)(R O E)
$$

En el proceso se generaron tres nuevos filtros. Primero, solo se seleccionaron las empresas que pagaron dividendos. Segundo, que no presentara una tasa de pago de dividendos superior al $100 \%$, debido a que se considera que quienes tienen una tasa de pago de dividendos superior al $100 \%$ de sus utilidades obedecen a una condición específica, que difícilmente se puede repetir de manera indeterminada. Y tercero, solo se analizaron compañías maduras en el mercado de valores, por lo tanto, no se consideraron los modelos de descuento de dividendos por etapas, lo cual nos llevó a determinar que la tasa de crecimiento de los dividendos de las compañías analizadas no puede superar la expectativa de crecimiento de la economía en el largo plazo.

La expectativa de crecimiento de la economía para los países de la Alianza del Pacífico se tomó del Fondo Monetario Internacional (2017, p. 72). También se tuvo en cuenta la actualización del Fondo Monetario Internacional - FMI (FMI, 2018).

Tasa de rentabilidad exigida a las acciones, teniendo en cuenta que,

Una abrumadora mayoría de las organizaciones (87 por ciento) usan el modelo de fijación de precios de activos de capital (CAPM) al estimar su costo de capital. CAPM es el método preferido para estimar el costo del capital independientemente del tamaño de la organización y el tipo de propiedad (AFP, 2011, p. 9). 
EI CAPM fue el modelo a través del cual se calculó la tasa de rendimiento exigida por los inversionistas. Para el cálculo del modelo, el beta fue estimado con el software Economática, al igual que la tasa libre de riesgo y el rendimiento del mercado; para estos dos últimos, en un análisis preliminar de la rentabilidad de mercado, se consideró en 107 meses el periodo más estable. El riesgo país, se tomó de la página del profesor Damodaran, quien reporta el ranking de Moody's anualmente.

Valor intrínseco, este valor se obtiene de la aplicación de la fórmula (2) a los datos mencionados.

Rendimiento a 1 año, en este se asume una tenencia definida donde:

$$
V 1=V o(1+g p)
$$

Por lo tanto, el rendimiento a 1 año estará determinado por:

$$
R=\frac{V 1+D 1}{P o}(6)
$$

Donde Po es el precio de cierre.

Rendimiento en tenencia de largo plazo (indefinida), se calculó con la siguiente fórmula:

$$
i *=\frac{D 1}{P o}+g p
$$

El paso final consistió en realizar una comparación del comportamiento de los índices MSCI (Morgan Stanley Capital International), para los países de la Alianza. Dichos índices miden el rendimiento de un conjunto de valores de renta variable, para un país determinado. "Los índices de acciones de $\mathrm{MSCl}$ se calcularon utilizando el concepto de promedio aritmético ponderado de Laspeyres junto con el concepto de encadenamiento» (MSCl, 2018, p. 5).

\section{RESULTADOS}

Las acciones consideradas en el estudio se encuentran representadas y ordenadas alfabéticamente en las siguientes Tablas 1, 2, 3 y 4.

De las 59 empresas en Colombia que reporta el software Economática, 15 pasaron los filtros (ver Tabla 1), realizados los cálculos a través del DDM, el $73.33 \%$ de ellas presenta sobrevaloración, es decir su valor intrínseco es superior a su valor de mercado.

Al calcular para las acciones de Colombia el exceso del valor de mercado con respecto al valor intrínseco, excluyendo la acción CONCONCRET, quien tiene un valor en exceso del $22.4 \%$, es posible determinar la alta sobrevaloración y el amplio intervalo entre el cual se mueven las empresas sobrevaloradas, las cuales se encuentran entre el $76.4 \%$ para CELSIA y el $364.3 \%$ para ECOPETROL. Por otra parte, la subvaloración no se presenta en grandes cifras ni en un amplio intervalo de distribución, pues la acción más subvalorada es PFAVAL con un $14.3 \%$ y la menos subvalorada es BOGOTA con un $8.2 \%$; si asumimos que el precio de las acciones es convergente a su valor intrínseco, 
las oportunidades de inversión por subvaloración, presentan menores expectativas de ganancia que las posibles pérdidas por compra de acciones sobrevaloradas.

Tabla 1. Valor intrínseco acciones Colombia

Table 1. Intrinsic value of stocks in Colombia

\begin{tabular}{llllll}
\hline \multicolumn{7}{c}{ Código } & $\begin{array}{c}\text { Precio de Cierre } \\
\left(\mathbf{P}_{0}\right) \\
\text { Abr 18 en } \\
\text { moneda original }\end{array}$ & $\begin{array}{c}\text { Dividendo por } \\
\text { Acción Abr } 18 \\
1 \text { años en } \\
\text { moneda original }\end{array}$ & $\begin{array}{c}\text { Utilidad por } \\
\text { Acción más } \\
\text { reciente en } \\
\text { moneda original } \\
\text { de } 12 \text { meses }\end{array}$ & $\begin{array}{c}\text { Valor intrínseco } \\
\left(\mathbf{V}_{0}\right)\end{array}$ & Resultado \\
\hline BOGOTA & $\$ 69.500,00$ & $\$ 2.860,00$ & $\$ 6.230,76$ & $\$ 75.687,45$ & subvalorada \\
\hline PFDAVVNDA & $\$ 31.360,00$ & $\$ 475,00$ & $\$ 2.870,84$ & $\$ 12.052,47$ & sobrevalorada \\
\hline BCOLOMBIA & $\$ 32.960,00$ & $\$ 712,80$ & $\$ 2.718,78$ & $\$ 18.341,49$ & sobrevalorada \\
\hline PFBCOLOM & $\$ 32.600,00$ & $\$ 712,80$ & $\$ 2.718,78$ & $\$ 18.345,28$ & sobrevalorada \\
\hline CELSIA & $\$ 4.690,00$ & $\$ 130,00$ & $\$ 201,56$ & $\$ 2.658,21$ & sobrevalorada \\
\hline ELCONDOR & $\$ 1.135,00$ & $\$ 48,99$ & $\$ 312,35$ & $\$ 1.301,37$ & subvalorada \\
\hline CONCONCRET & $\$ 950,00$ & $\$ 29,10$ & $\$ 68,95$ & $\$ 776,42$ & sobrevalorada \\
\hline ECOPETROL & $\$ 2.740,00$ & $\$ 23,00$ & $\$ 161,02$ & $\$ 590,09$ & sobrevalorada \\
\hline EXITO & $\$ 16.040,00$ & $\$ 145,92$ & $\$ 1.905,83$ & $\$ 3.700,22$ & sobrevalorada \\
\hline GRUPOARGOS & $\$ 19.360,00$ & $\$ 310,00$ & $\$ 707,66$ & $\$ 6.478,82$ & sobrevalorada \\
\hline PFGRUPOARG & $\$ 16.980,00$ & $\$ 310,00$ & $\$ 707,66$ & $\$ 6.519,23$ & sobrevalorada \\
\hline GRUPOAVAL & $\$ 1.215,00$ & $\$ 53,90$ & $\$ 101,91$ & $\$ 1.417,74$ & subvalorada \\
\hline PFAVAL & $\$ 1.215,00$ & $\$ 53,90$ & $\$ 101,91$ & $\$ 1.417,98$ & subvalorada \\
\hline ISA & $\$ 13.880,00$ & $\$ 288,00$ & $\$ 1.298,15$ & $\$ 7.365,13$ & sobrevalorada \\
\hline NUTRESA & $\$ 26.240,00$ & $\$ 534,00$ & $\$ 913,25$ & $\$ 11.157,16$ & sobrevalorada \\
\hline & & Fuente: (Economática, 2018). & &
\end{tabular}

De las 165 empresas en México que reporta el software Economática, 46 pasaron los filtros (ver Tabla 2), realizados los cálculos a través del DDM, el $89.13 \%$ de ellas presenta sobrevaloración.

Tabla 2. Valor intrínseco acciones México

Table 2. Intrinsic value of stocks in Mexico

\begin{tabular}{|c|c|c|c|c|c|}
\hline Código & $\begin{array}{c}\text { Precio de Cierre } \\
\left(\mathrm{P}_{0}\right) \\
05 \mathrm{Abr} 18 \\
\text { en moneda } \\
\text { original }\end{array}$ & $\begin{array}{l}\text { Dividendo por } \\
\text { Acción } \\
05 \mathrm{Abr} 18 \\
1 \text { años } \\
\text { en moneda original }\end{array}$ & $\begin{array}{l}\text { Utilidad por Acción } \\
\text { más reciente } \\
\text { en moneda original } \\
\text { de } 12 \text { meses }\end{array}$ & $\begin{array}{l}\text { Valor intrínseco } \\
\qquad\left(\mathrm{V}_{0}\right)\end{array}$ & Resultado \\
\hline ALSEA & $\$ 65,67$ & $\$ 0,68$ & $\$ 1,31$ & $\$ 9,70$ & sobrevalora \\
\hline AMXA & $\$ 17,27$ & $\$ 0,30$ & $\$ 0,44$ & $\$ 4,30$ & sobrevalora \\
\hline ARA & $\$ 7,38$ & $\$ 0,08$ & $\$ 0,70$ & $\$ 1,24$ & sobrevalora \\
\hline$A C$ & $\$ 126,27$ & $\$ 2,00$ & $\$ 7,48$ & $\$ 28,04$ & sobrevalora \\
\hline ASURB & $\$ 311,69$ & $\$ 6,16$ & $\$ 19,45$ & $\$ 89,29$ & sobrevalora \\
\hline AUTLANB & $\$ 20,00$ & $\$ 0,22$ & $\$ 4,70$ & $\$ 3,38$ & sobrevalora \\
\hline $\mathrm{BACHOCO}$ & $\$ 96,87$ & $\$ 1,30$ & $\$ 8,07$ & $\$ 19,37$ & sobrevalora \\
\hline BIMBOA & $\$ 40,94$ & $\$ 0,29$ & $\$ 0,98$ & $\$ 4,16$ & sobrevalora \\
\hline BOLSAA & $\$ 36,20$ & $\$ 1,25$ & $\$ 1,96$ & $\$ 17,19$ & sobrevalora \\
\hline GCC & $\$ 97,44$ & $\$ 0,62$ & $\$ 4,60$ & $\$ 9,11$ & sobrevalora \\
\hline CHDRAUIB & $\$ 39,07$ & $\$ 0,33$ & $\$ 2,16$ & $\$ 4,60$ & sobrevalora \\
\hline CMOCTEZ & $\$ 74,29$ & $\$ 5,00$ & $\$ 5,36$ & $\$ 74,24$ & sobrevalora \\
\hline CULTIBAB & $\$ 15,32$ & $\$ 0,29$ & $\$ 12,99$ & $\$ 4,10$ & sobrevalora \\
\hline CYDSASAA & $\$ 31,37$ & $\$ 0,31$ & $\$ 0,81$ & $\$ 4,64$ & sobrevalora \\
\hline
\end{tabular}




\begin{tabular}{llllll}
\hline DANHOS1 & $\$ 30,34$ & $\$ 2,28$ & $\$ 3,67$ & $\$ 33,84$ & subvalorada \\
\hline ELEKTRA & $\$ 521,32$ & $\$ 3,50$ & $\$ 66,38$ & $\$ 49,44$ & sobrevalora \\
\hline FSHOP13 & $\$ 10,87$ & $\$ 0,85$ & $\$ 3,52$ & $\$ 12,54$ & subvalorada \\
\hline FIBRAMQ1 & $\$ 20,55$ & $\$ 1,50$ & $\$ 2,28$ & $\$ 21,58$ & subvalorada \\
\hline FUNO11 & $\$ 29,34$ & $\$ 2,05$ & $\$ 3,60$ & $\$ 30,21$ & subvalorada \\
\hline GCARSOA1 & $\$ 65,41$ & $\$ 0,90$ & $\$ 4,43$ & $\$ 12,80$ & sobrevalora \\
\hline GENTERA & $\$ 14,19$ & $\$ 1,16$ & $\$ 1,77$ & $\$ 16,20$ & subvalorada \\
\hline GFNORTE & $\$ 115,24$ & $\$ 2,78$ & $\$ 8,62$ & $\$ 39,28$ & sobrevalora \\
\hline GFINBURO & $\$ 31,03$ & $\$ 0,46$ & $\$ 3,00$ & $\$ 6,48$ & sobrevalora \\
\hline GFINTERO & $\$ 88,74$ & $\$ 4,28$ & $\$ 10,89$ & $\$ 61,95$ & sobrevalora \\
\hline GFREGIOO & $\$ 114,88$ & $\$ 4,30$ & $\$ 8,41$ & $\$ 61,75$ & sobrevalora \\
\hline GISSAA & $\$ 28,71$ & $\$ 1,05$ & $\$ 3,13$ & $\$ 15,27$ & sobrevalora \\
\hline GMEXICOB & $\$ 62,47$ & $\$ 1,50$ & $\$ 4,01$ & $\$ 21,50$ & sobrevalora \\
\hline GRUMAB & $\$ 216,52$ & $\$ 3,70$ & $\$ 14,37$ & $\$ 53,79$ & sobrevalora \\
\hline GSANBOR & $\$ 20,18$ & $\$ 0,88$ & $\$ 1,72$ & $\$ 13,13$ & sobrevalora \\
\hline HERDEZ & $\$ 46,20$ & $\$ 1,35$ & $\$ 2,43$ & $\$ 19,50$ & sobrevalora \\
\hline IENOVA & $\$ 92,34$ & $\$ 0,13$ & $\$ 4,54$ & $\$ 1,89$ & sobrevalora \\
\hline LALAB & $\$ 24,77$ & $\$ 0,62$ & $\$ 1,31$ & $\$ 8,86$ & sobrevalora \\
\hline LIVEPOLC- & $\$ 139,11$ & $\$ 0,96$ & $\$ 7,37$ & $\$ 13,75$ & sobrevalora \\
\hline MEDICAB & $\$ 39,80$ & $\$ 0,70$ & $\$ 1,85$ & $\$ 10,51$ & sobrevalora \\
\hline MEGACPO & $\$ 83,10$ & $\$ 1,62$ & $\$ 2,41$ & $\$ 24,19$ & sobrevalora \\
\hline MEXCHEM & $\$ 57,99$ & $\$ 1,02$ & $\$ 1,82$ & $\$ 14,18$ & sobrevalora \\
\hline OHLMEX & $\$ 33,44$ & $\$ 0,40$ & $\$ 6,12$ & $\$ 5,67$ & sobrevalora \\
\hline OMAB & $\$ 91,49$ & $\$ 4,00$ & $\$ 5,40$ & $\$ 56,82$ & sobrevalora \\
\hline PE\&OLES & $\$ 396,46$ & $\$ 10,88$ & $\$ 27,40$ & $\$ 154,50$ & sobrevalora \\
\hline PINFRA & $\$ 186,28$ & $\$ 1,88$ & $\$ 10,32$ & $\$ 26,96$ & sobrevalora \\
\hline Q & $\$ 51,66$ & $\$ 0,60$ & $\$ 4,57$ & $\$ 8,96$ & sobrevalora \\
\hline RASSINIA & $\$ 39,67$ & $\$ 2,00$ & $\$ 4,61$ & $\$ 29,06$ & sobrevalora \\
\hline BSMXB & $\$ 27,37$ & $\$ 1,58$ & $\$ 2,63$ & $\$ 21,64$ & sobrevalora \\
\hline TLEVISACP & $\$ 64,05$ & $\$ 0,00$ & $\$ 0,01$ & $\$ 0,04$ & sobrevalora \\
\hline VITROA & $\$ 60,67$ & $\$ 0,06$ & $\$ 7,59$ & $\$ 0,95$ & sobrevalora \\
\hline WALMEX & $\$ 48,64$ & $\$ 0,64$ & $\$ 2,28$ & $\$ 8,97$ & sobrevalora \\
\hline & & Fuente: (Economática, 2018$)$ & & \\
\hline
\end{tabular}

El fenómeno analizado para Colombia de amplios intervalos de sobre valoración y cortos intervalos de subvaloración se repite en México, sin embargo, eliminando del análisis casos extremos como la sobre valoración de TLEVISACP, se identifica que el intervalo superior de sobre valoración para las acciones mexicanas, presenta mayores excesos que el caso colombiano, teniendo en el grupo grandes sobrevaloraciones para acciones como: VITROA (6286 \%), IENOVA (4785\%), GCC (969\%), ELEKTRA (954 \%), LIVEPOLC-1 (911 \%) y BIMBOA (884 \%).

De las 230 empresas en Perú que reporta el software Economática, 11 pasaron los filtros (ver Tabla 3), realizados los cálculos a través del DDM, el 63.64 \% de ellas presenta sobrevaloración.

La forma de distribución de la sobrevaloración en las acciones de Perú, presenta un caso extremo con la acción de BVN (1622\%), pero en las demás acciones muestra menores valores y menores intervalos de sobrevaloración que en Colombia y México, adicionalmente existen casos en los cuales la subvaloración se presenta en grandes cifras, por lo tanto de acuerdo con la hipótesis de convergencia al valor intrínseco, las oportunidades de inversión por subvaloración, presentan mayores expectativas de ganancias que en los demás países de la Alianza del Pacífico; adicionalmente la muestra del Perú posee la mayor participación relativa con un 34,6\%, de las acciones subvaloradas, 
lo cual podría representar una señal de oportunidades de lograr mayor rentabilidad al invertir en Perú.

Tabla 3. Valor intrínseco acciones Perú

Table 3. Intrinsic value of stocks in Peru

\begin{tabular}{llclll}
\hline Código & $\begin{array}{c}\text { Precio de } \\
\text { Cierre }\left(\mathrm{P}_{0}\right) \\
\text { 05 } \mathrm{Abr} 18 \\
\text { en moneda } \\
\text { original }\end{array}$ & $\begin{array}{c}\text { Dividendo por } \\
\text { Acción } \\
\text { 05 Abr } 18 \\
1 \text { años } \\
\text { en moneda } \\
\text { original }\end{array}$ & $\begin{array}{c}\text { Utilidad por } \\
\text { Acción } \\
\text { más reciente } \\
\text { en moneda } \\
\text { original } \\
\text { de } 12 \text { meses }\end{array}$ & $\begin{array}{c}\text { Valor intrínseco } \\
\left(V_{0}\right)\end{array}$ & Resultado \\
\hline ALICORC1 & $\$ 11,07$ & $\$ 0,14$ & $\$ 0,53$ & $\$ 4,34$ & sobrevalorada \\
\hline CONTINC1 & $\$ 4,50$ & $\$ 0,15$ & $\$ 0,28$ & $\$ 4,10$ & sobrevalorada \\
\hline BVN & $\$ 15,85$ & $\$ 0,09$ & $\$ 0,77$ & $\$ 0,92$ & sobrevalorada \\
\hline CORAREC1 & $\$ 0,87$ & $\$ 0,04$ & $\$ 0,12$ & $\$ 1,01$ & subvalorada \\
\hline CORAREI & $\$ 0,75$ & $\$ 0,04$ & $\$ 0,12$ & $\$ 0,72$ & sobrevalorada \\
\hline FERREYC1 & $\$ 2,53$ & $\$ 0,13$ & $\$ 0,27$ & $\$ 3,28$ & subvalorada \\
\hline INRETC1 & $\$ 22,25$ & $\$ 0,19$ & $\$ 2,92$ & $\$ 7,26$ & sobrevalorada \\
\hline IFS & $\$ 40,12$ & $\$ 1,30$ & $\$ 7,41$ & $\$ 38,21$ & sobrevalorada \\
\hline LUSURC1 & $\$ 12,00$ & $\$ 0,62$ & $\$ 0,96$ & $\$ 33,01$ & subvalorada \\
\hline LUISAI1 & $\$ 220,00$ & $\$ 28,50$ & $\$ 36,43$ & $\$ 2,390,02$ & subvalorada \\
\hline UNACEMC1 & $\$ 2,90$ & $\$ 0,05$ & $\$ 0,15$ & $\$ 1,01$ & sobrevalorada \\
\hline
\end{tabular}

De las 240 empresas en Chile que reporta el software Economática, 61 pasaron los filtros (ver Tabla 4), realizados los cálculos a través del DDM, el $90.16 \%$ de ellas presenta sobrevaloración.

Tabla 4. Valor intrínseco acciones Chile

Table 4. Intrinsic value of stocks in Chile

\begin{tabular}{|c|c|c|c|c|c|}
\hline Código & $\begin{array}{c}\text { Precio de Cierre } \\
\left(\mathrm{P}_{0}\right) \\
05 \mathrm{Abr} 18 \\
\text { en moneda } \\
\text { original }\end{array}$ & $\begin{array}{l}\text { Dividendo por } \\
\text { Acción } \\
05 \mathrm{Abr} 18 \\
1 \text { años } \\
\text { en moneda } \\
\text { original }\end{array}$ & $\begin{array}{l}\text { Utilidad por } \\
\text { Acción } \\
\text { más reciente } \\
\text { en moneda } \\
\text { original } \\
\text { de } 12 \text { meses }\end{array}$ & $\begin{array}{l}\text { Valor intrínseco } \\
\qquad\left(\mathrm{V}_{0}\right)\end{array}$ & Resultado \\
\hline HABITAT & $\$ 1.020,10$ & $\$ 59,00$ & $\$ 104,08$ & $\$ 1.281,35$ & subvalorada \\
\hline AGUAS-A & $\$ 394,38$ & $\$ 22,12$ & $\$ 22,82$ & $\$ 378,35$ & sobrevalorada \\
\hline ANTARCHILE & $\$ 11.018,00$ & $\$ 195,17$ & $\$ 537,94$ & $\$ 3.629,91$ & sobrevalorada \\
\hline CHILE & $\$ 101,20$ & $\$ 3,15$ & $\$ 5,90$ & $\$ 73,00$ & sobrevalorada \\
\hline $\mathrm{BCl}$ & $\$ 44.995,00$ & $\$ 1.050,00$ & $\$ 3.005,75$ & $\$ 20.747,85$ & sobrevalorada \\
\hline ITAUCORP & $\$ 6,00$ & $\$ 0,04$ & $\$ 0,11$ & $\$ 0,60$ & sobrevalorada \\
\hline BSANTANDER & $\$ 51,76$ & $\$ 1,75$ & $\$ 3,00$ & $\$ 30,96$ & sobrevalorada \\
\hline BANMEDICA & $\$ 2.150,00$ & $\$ 36,00$ & $\$ 75,90$ & $\$ 750,39$ & sobrevalorada \\
\hline BANVIDA & $\$ 488,79$ & $\$ 17,50$ & $\$ 58,57$ & $\$ 381,14$ & sobrevalorada \\
\hline BESALCO & $\$ 702,29$ & $\$ 8,00$ & $\$ 17,02$ & $\$ 78,59$ & sobrevalorada \\
\hline CENCOSUD & $\$ 1.834,20$ & $\$ 30,00$ & $\$ 153,71$ & $\$ 513,37$ & sobrevalorada \\
\hline CINTAC & $\$ 399,00$ & $\$ 10,09$ & $\$ 21,91$ & $\$ 238,78$ & sobrevalorada \\
\hline EMBONOR-B & $\$ 1.750,00$ & $\$ 60,00$ & $\$ 82,38$ & $\$ 1.253,02$ & sobrevalorada \\
\hline COLBUN & $\$ 143,69$ & $\$ 4,24$ & $\$ 9,51$ & $\$ 92,97$ & sobrevalorada \\
\hline $\mathrm{CCU}$ & $\$ 8.821,10$ & $\$ 180,32$ & $\$ 350,76$ & $\$ 3.809,52$ & sobrevalorada \\
\hline INTEROCEAN & $\$ 73,45$ & $\$ 3,65$ & $\$ 6,17$ & $\$ 96,70$ & subvalorada \\
\hline
\end{tabular}




\begin{tabular}{|c|c|c|c|c|c|}
\hline ANDINA-A & $\$ 2.650,00$ & $\$ 59,50$ & $\$ 124,49$ & $\$ 1.248,77$ & sobrevalorada \\
\hline ANDINA-B & $\$ 2.988,00$ & $\$ 65,45$ & $\$ 124,49$ & $\$ 1.235,17$ & sobrevalorada \\
\hline ENTEL & $\$ 7.123,40$ & $\$ 34,00$ & $\$ 143,73$ & $\$ 662,70$ & sobrevalorada \\
\hline CMPC & $\$ 2.312,20$ & $\$ 1,26$ & $\$ 25,43$ & $\$ 17,16$ & sobrevalorada \\
\hline COPEC & $\$ 9.774,20$ & $\$ 135,64$ & $\$ 302,59$ & $\$ 2.491,56$ & sobrevalorada \\
\hline HITES & $\$ 625,65$ & $\$ 21,33$ & $\$ 35,47$ & $\$ 287,14$ & sobrevalorada \\
\hline ENELAM & $\$ 143,01$ & $\$ 2,99$ & $\$ 7,54$ & $\$ 59,96$ & sobrevalorada \\
\hline ENELGXCH & $\$ 499,58$ & $\$ 26,50$ & $\$ 51,02$ & $\$ 542,82$ & subvalorada \\
\hline $\mathrm{ECL}$ & $\$ 1.287,20$ & $\$ 8,19$ & $\$ 58,91$ & $\$ 152,03$ & sobrevalorada \\
\hline PASUR & $\$ 7.900,00$ & $\$ 60,00$ & $\$ 266,70$ & $\$ 1.601,82$ & sobrevalorada \\
\hline FORUS & $\$ 2.400,00$ & $\$ 40,85$ & $\$ 104,44$ & $\$ 711,05$ & sobrevalorada \\
\hline GASCO & $\$ 2.506,80$ & $\$ 68,00$ & $\$ 167,95$ & $\$ 2.228,73$ & sobrevalorada \\
\hline SECURITY & $\$ 310,01$ & $\$ 12,10$ & $\$ 21,62$ & $\$ 254,38$ & sobrevalorada \\
\hline $\mathrm{HF}$ & $\$ 2.000,00$ & $\$ 19,93$ & $\$ 37,52$ & $\$ 415,37$ & sobrevalorada \\
\hline INVERCAP & $\$ 2.197,50$ & $\$ 16,90$ & $\$ 149,70$ & $\$ 220,10$ & sobrevalorada \\
\hline IAM & $\$ 1.112,10$ & $\$ 66,66$ & $\$ 68,72$ & $\$ 992,00$ & sobrevalorada \\
\hline ILC & $\$ 11.825,00$ & $\$ 600,00$ & $\$ 1.293,78$ & $\$ 10.320,10$ & sobrevalorada \\
\hline LTM & $\$ 9.535,00$ & $\$ 23,00$ & $\$ 157,56$ & $\$ 293,95$ & sobrevalorada \\
\hline MINERA & $\$ 16.000,00$ & $\$ 300,00$ & $\$ 934,56$ & $\$ 10.276,99$ & sobrevalorada \\
\hline MOLYMET & $\$ 8.600,00$ & $\$ 207,76$ & $\$ 360,11$ & $\$ 4.980,71$ & sobrevalorada \\
\hline MULTIFOODS & $\$ 293,26$ & $\$ 4,54$ & $\$ 29,48$ & $\$ 75,38$ & sobrevalorada \\
\hline NITRATOS & $\$ 2,44$ & $\$ 0,03$ & $\$ 0,09$ & $\$ 0,31$ & sobrevalorada \\
\hline NORTEGRAN & $\$ 6,52$ & $\$ 0,09$ & $\$ 0,30$ & $\$ 0,92$ & sobrevalorada \\
\hline PARAUCO & $\$ 1.852,40$ & $\$ 32,00$ & $\$ 99,55$ & $\$ 759,24$ & sobrevalorada \\
\hline PAZ & $\$ 920,00$ & $\$ 29,00$ & $\$ 76,96$ & $\$ 403,45$ & sobrevalorada \\
\hline VENTANAS & $\$ 168,90$ & $\$ 8,97$ & $\$ 10,26$ & $\$ 237,80$ & subvalorada \\
\hline QUINENCO & $\$ 2.030,00$ & $\$ 31,92$ & $\$ 65,40$ & $\$ 789,35$ & sobrevalorada \\
\hline RIPLEY & $\$ 640,00$ & $\$ 10,95$ & $\$ 44,34$ & $\$ 174,21$ & sobrevalorada \\
\hline FALABELLA & $\$ 5.837,80$ & $\$ 80,00$ & $\$ 209,32$ & $\$ 1.683,50$ & sobrevalorada \\
\hline SALFACORP & $\$ 1.146,00$ & $\$ 16,78$ & $\$ 54,95$ & $\$ 198,25$ & sobrevalorada \\
\hline SK & $\$ 1.206,00$ & $\$ 21,58$ & $\$ 47,72$ & $\$ 364,37$ & sobrevalorada \\
\hline SM-CHILE B & $\$ 321,09$ & $\$ 3,16$ & $\$ 7,24$ & $\$ 70,76$ & sobrevalorada \\
\hline SM-CHILE D & $\$ 304,10$ & $\$ 3,16$ & $\$ 7,24$ & $\$ 75,98$ & sobrevalorada \\
\hline SM-CHILE E & $\$ 92,00$ & $\$ 3,16$ & $\$ 7,24$ & $\$ 87,01$ & sobrevalorada \\
\hline ORO BLANCO & $\$ 6,50$ & $\$ 0,08$ & $\$ 0,28$ & $\$ 1,14$ & sobrevalorada \\
\hline SMSAAM & $\$ 63,01$ & $\$ 2,00$ & $\$ 3,82$ & $\$ 36,87$ & sobrevalorada \\
\hline PUCOBRE-A & $\$ 3.900,00$ & $\$ 65,42$ & $\$ 178,20$ & $\$ 1.739,92$ & sobrevalorada \\
\hline SQM-B & $\$ 31.232,00$ & $\$ 921,22$ & $\$ 999,74$ & $\$ 10.009,01$ & sobrevalorada \\
\hline SOCOVESA & $\$ 380,58$ & $\$ 11,60$ & $\$ 32,73$ & $\$ 159,29$ & sobrevalorada \\
\hline SONDA & $\$ 1.200,10$ & $\$ 8,71$ & $\$ 74,50$ & $\$ 165,13$ & sobrevalorada \\
\hline CONCHATORO & $\$ 1.325,00$ & $\$ 27,30$ & $\$ 66,36$ & $\$ 761,67$ & sobrevalorada \\
\hline VSPT & $\$ 7,10$ & $\$ 0,35$ & $\$ 0,44$ & $\$ 7,79$ & subvalorada \\
\hline WATTS & $\$ 1.240,00$ & $\$ 32,00$ & $\$ 47,05$ & $\$ 833,73$ & sobrevalorada \\
\hline ZOFRI & $\$ 710,87$ & $\$ 37,53$ & $\$ 54,42$ & $\$ 1.372,83$ & subvalorada \\
\hline
\end{tabular}

Eliminando el caso extremo de sobre de sobrevaloración para las acciones de chile (CMPC=13374 \%), se observa que Chile es el segundo país de la Alianza del Pacífico que posee un intervalo superior con cifras excesivas en la sobrevaloración de acciones, adicionalmente es el país que inicia con menores valores en el límite de inferior de su intervalo de sobrevaloración. Por otra parte, la participación relativa de las acciones subvaloradas correspondiente a un $10 \%$, es menor que en las demás muestras tomada para los países de la Alianza del Pacífico. 
Al comparar los índices MSCI de los países pertenecientes a la Alianza del Pacífico y el índice global de los países emergentes MSCl, Emerging Markets Index (ver Figura 1), se puede observar el comportamiento alcista de los índices para los últimos 2 años.

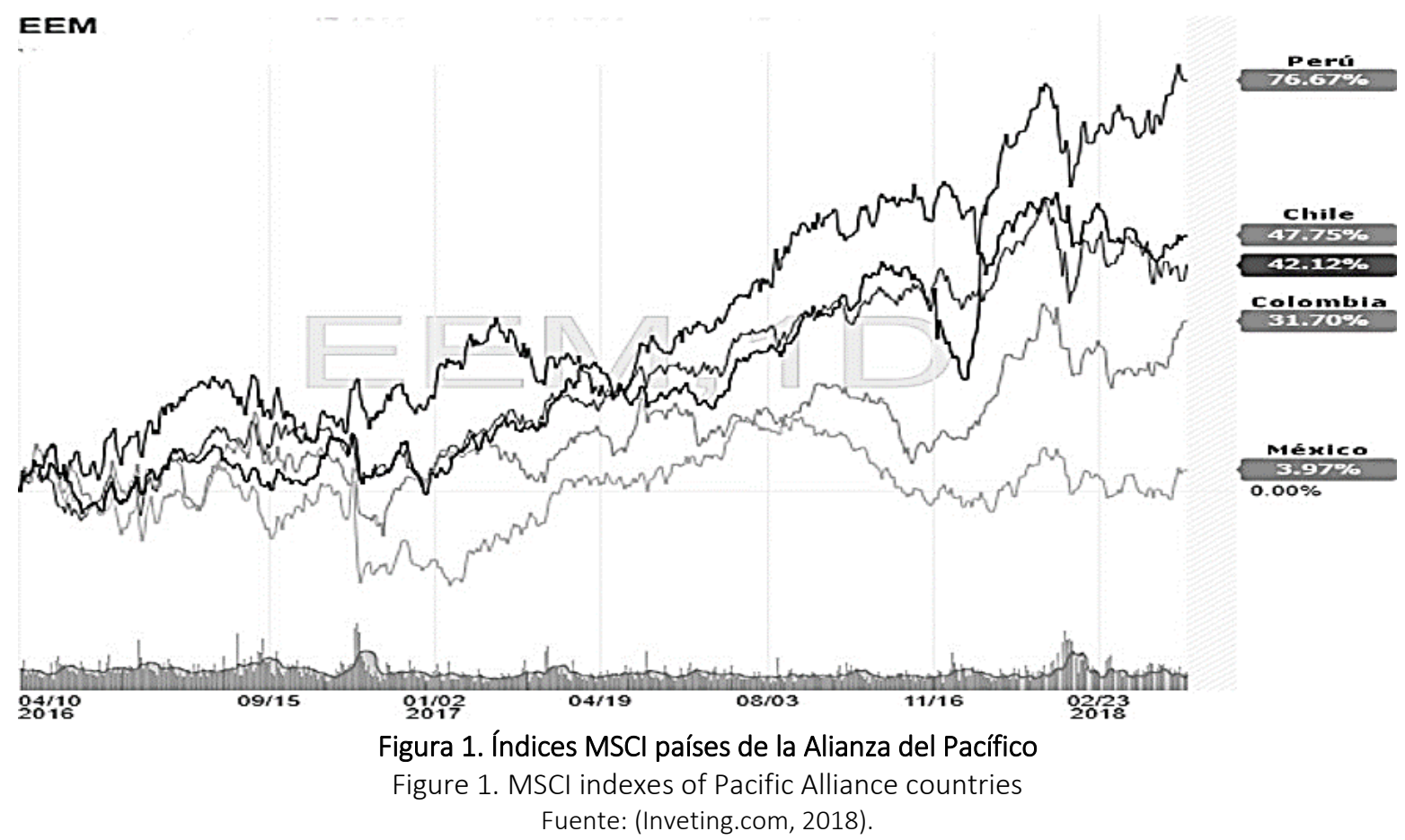

Las empresas subvaloradas en el mercado de renta variable de la Alianza del Pacífico, se relacionan en la Tabla 5.

Tabla 5. Empresas subvaloradas en el mercado de la AdP

Table 5. Undervalued companies in the Pacific Alliance market

\begin{tabular}{llllll}
\hline \multicolumn{1}{c}{ Nombre } & \multicolumn{1}{c}{$\begin{array}{c}\text { Precio de } \\
\text { País }\end{array}$} & $\begin{array}{c}\text { Cierre }\left(\mathrm{P}_{0}\right) \\
\mathbf{0 5} \text { abril } 2018\end{array}$ & $\begin{array}{c}\text { Valor } \\
\text { ROE }\end{array}$ & $\begin{array}{c}\text { Rendimiento } \\
\text { intrínseco } \\
\left(\mathrm{V}_{0}\right)\end{array}$ & $\begin{array}{c}\text { en tenencia de } \\
\text { largo plazo } \\
\text { (indefinida) }\end{array}$ \\
\hline Colombia & Banco Bogotá & $\$ 69.500,00$ & 13,3 & $\$ 75.687,45$ & $7,12 \%$ \\
\hline Colombia & Construcciones El Condor S.A. & $\$ 1.135,00$ & 22,3 & $\$ 1.301,37$ & $7,32 \%$ \\
\hline Colombia & Grupo Aval Ac Va & $\$ 1.215,00$ & 8,4 & $\$ 1.417,74$ & $7,44 \%$ \\
\hline Colombia & Grupo Aval Ac Va & $\$ 1.215,00$ & 8,4 & $\$ 1.417,98$ & $7,44 \%$ \\
\hline México & Danhos & $\$ 30,34$ & 9,99 & $\$ 33,84$ & $10 \%$ \\
\hline México & Fibra Shop & $\$ 10,87$ & 18,07 & $\$ 12,54$ & $10 \%$ \\
\hline México & Fibramq & $\$ 20,55$ & 6,81 & $\$ 21,58$ & $10 \%$ \\
\hline México & Funo & $\$ 29,34$ & 9,90 & $\$ 30,21$ & $9 \%$ \\
\hline México & Gentera & $\$ 14,19$ & 17,97 & $\$ 16,20$ & $10 \%$ \\
\hline Perú & Corp. Aceros Arequipa & $\$ 0,87$ & 7,06 & $\$ 1,01$ & $8 \%$ \\
\hline Perú & Ferreycorp S.A.A. & $\$ 2,53$ & 13,91 & $\$ 3,28$ & $9 \%$ \\
\hline Perú & Luz del Sur S.A. & $\$ 12,00$ & 19,44 & $\$ 33,01$ & $9 \%$ \\
\hline Perú & Santa Luisa & $\$ 220,00$ & 48,33 & $\$ 2.390,02$ & $17 \%$ \\
\hline Chile & A.F.P. Hábitat S.A. & $\$ 1.020,10$ & 33,4 & $\$ 1.281,35$ & $9 \%$ \\
\hline Chile & Compañía Marítima Chilena S.A. & $\$ 73,45$ & 6,9 & $\$ 96,70$ & $8 \%$ \\
\hline
\end{tabular}




\begin{tabular}{llllll}
\hline Chile & Enel Generación Chile S.A. & $\$ 499,58$ & 24,6 & $\$ 542,82$ & $8 \%$ \\
\hline Chile & Puerto Ventanas S.A. & $\$ 168,90$ & 12,0 & $\$ 237,80$ & $7 \%$ \\
\hline Chile & Viña San Pedro Tarapacá S.A. & $\$ 7,10$ & 8,3 & $\$ 7,79$ & $7 \%$ \\
\hline Chile & Zona Franca De Iquique S.A. & $\$ 710,87$ & 29,7 & $\$ 1.372,83$ & $8 \%$ \\
\hline
\end{tabular}

Fuente: (Economática, 2018).

Las empresas subvaloradas de la Alianza del Pacífico presentan un rendimiento promedio sobre el patrimonio del 16.77 \%, entre estas encontramos Compañía Minera Santa Luisa en Perú la cual se caracteriza por ser una de las compañías más rentables de la Alianza. Por otra parte, para estas acciones calculamos un rendimiento promedio en tenencia de largo plazo del $8.86 \%$.

El promedio de ROE y el promedio de la tasa de retención de utilidades, para los países de la Alianza del Pacífico, se detallan en la Figura 2.

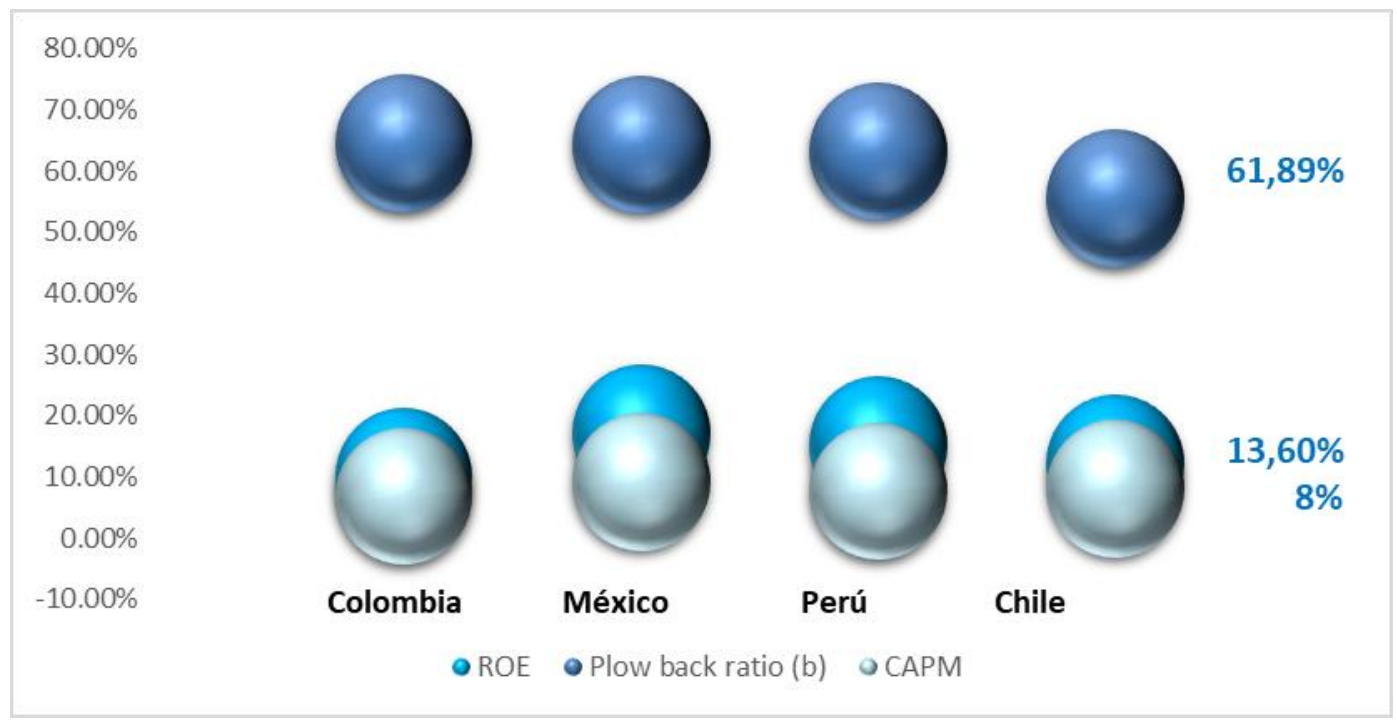

Figura 2. Promedio de ROE y Plowback ratio

Figure 2. Average ROE and plowback ratio

Fuente: (Economática, 2018).

A partir de los cálculos realizados, se puede observar una considerable relación (Figura 2), en el rendimiento del patrimonio (ROE), la tasa de retención de utilidades (Plow back ratio) y el modelo de determinación del precio de los activos de capital Capital Asset Pricing Model (CAPM) para los países de la Alianza del Pacífico.

Los promedios de sobrevaloración, ROE y el crecimiento en el índice $\mathrm{MSCl}$ de los países pertenecientes a la Alianza del Pacífico, y el índice global de los países emergentes MSCl Emerging Markets Index, son presentados en la Figura 3. 


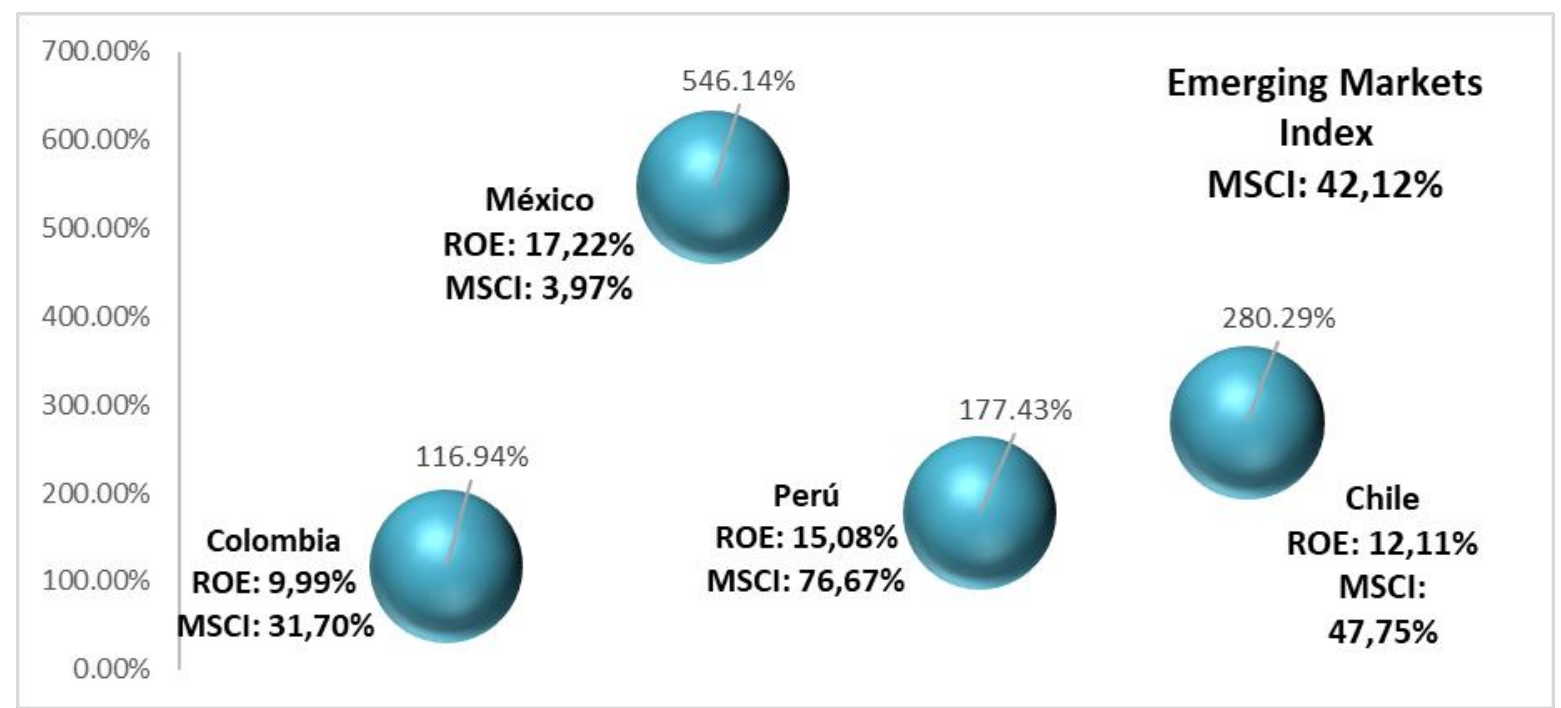

Figura 3. Promedio de sobrevaloración y ROE

Figure 3. Average overvaluation and ROE

Fuente: (Economática, 2018).

\section{DISCUSIÓN}

La presente investigación tuvo como propósito analizar las oportunidades de inversión en los países pertenecientes a la Alianza del Pacífico, usando el modelo de descuento de dividendos. De la muestra inicial de 694 acciones, solo se seleccionaron 132, debido a que la gran mayoría de estas no reportaron pago de dividendos, requisito indispensable para realizar la valoración, de ahí se puede deducir que el modelo no es aplicable en todos los casos, ya que depende de la capacidad de la empresa para producir utilidades y de las políticas de distribución de las mismas.

En los resultados obtenidos se encuentra que 116 de las 132 acciones en estudio presenta sobrevaloración, estos datos indican que los dividendos podrían no estar mostrando de manera eficiente el valor de las compañías o la tasa de descuento aplicado es superior a la que realmente acepta el mercado.

Por otro lado, al analizar los índices MSCl llama la atención el comportamiento del índice de México, que presenta una alta volatilidad y el menor crecimiento en el periodo de estudio (3.97 \%). Adicionalmente, al analizar los datos recolectados se encontró que el rendimiento sobre el patrimonio, así como la tasa de retención de utilidades, son similares en los países de la Alianza del Pacífico, por cual, encontrar marcadas diferencias en los promedios de sobrevaloración para las acciones de México, convierte al mercado de acciones de este país en un candidato para futuras investigaciones sobre análisis fundamental.

\section{CONCLUSIONES}

Considerando que con la integración financiera se constituye uno de los ejes centrales de la Alianza del Pacífico, y teniendo en cuenta la aplicación de una metodología estándar para la valoración de acciones por medio del DDM, es posible identificar importantes oportunidades de inversión para las 
empresas que se encuentran subvaloradas, a pesar de que se observó una sobrevaloración en la mayoría de las 132 acciones estudiadas. Adicionalmente, podrían existir mayores oportunidades de inversión para las acciones que cotizan en la bolsa de valores de Colombia, puesto que cuenta con la menor sobrevaloración, lo que en esencia debería llevar a un movimiento de capital de los demás países hacia las empresas en Colombia.

Por otra parte, el ROE promedio por país (Fig. 3), fue: México (17.22 \%), Perú (15.08\%), Chile (12.11\%) y Colombia (9.99\%), estos datos nos muestran a México como una de las economías más rentables de la Alianza del Pacífico; sin embargo, también se destaca por presentar el promedio más alto de sobrevaloración (546.14 \%), una alta volatilidad y el menor crecimiento de la Alianza en el índice $\mathrm{MSCl}$ (3.97\%) para los dos últimos años; estas señales nos permiten lanzar la hipótesis de que en México se estaría presentando una «burbuja de activos», lo que lo postula como un buen candidato para posteriores investigaciones.

Así mismo, se encontró una relación en los promedios de rentabilidad sobre el patrimonio, la tasa de descuento y la tasa de retención de utilidades (Fig. 2), para los países de la Alianza del Pacífico. Llama la atención las marcadas diferencias en los promedios de sobrevaloración (Fig. 3), ya que el modelo de descuento de dividendos depende de la tasa de retención de utilidades y la tasa de descuento, y esas tasas son más o menos similares en estos países (Fig. 2).

De manera adicional, a pesar de que el modelo de descuento de dividendos es una metodología de valoración ampliamente empleada y sirve como referente de las oportunidades de inversión en las empresas pertenecientes a la Alianza, los dividendos podrían no representar una expectativa clara sobre el valor de la compañía, por lo tanto, en la presente investigación el modelo de descuento de dividendos no estaría facultado para mostrar de manera eficiente el valor de todas las compañías, aunque también es posible que la ineficiencia resulte de la metodología usada para determinar la tasa de descuento, generando una tasa descuento mayor a la que implícitamente acepta el mercado.

Finalmente, como trabajo futuro, se podría repetir el estudio en 10 años para determinar cómo cambia la situación de las compañías con el desarrollo de la Alianza del Pacífico, realizar el mismo el ejercicio en otras alianzas y continuar con la validación del modelo en otros escenarios. Adicionalmente, se podría validar la hipótesis de ineficiencias en los dividendos o la metodología para la tasa de descuento.

\section{REFERENCIAS}

Acuerdo Marco de la Alianza del Pacífico. (2012). Acuerdo marco de la Alianza del Pacífico. Recuperado de https://www.cancilleria.gov.co/sites/default/files/alianza-delpacifico/acuerdo marco alianza del pacifico 06 jun 2012 mejorado.pdf

Anand, M. (2002). Corporate finance practices in India: A survey. Vikalpa, 27(4), 29-56. https://doi.org/10.1177/0256090920020404

Association for Financial Professionals, AFP (2011). Current Trends in Estimating and Applying the Cost of Capital. Recuperado de 
http://business.baylor.edu/don cunningham/How Firms Estimate Cost of Capital (20 11).pdf

Berk, J. \& Demarzo. (2008). Finanzas Corporativas. México D. F., México: Pearson Educación.

Charumathi, B., \& Suraj, E. (2014). The Reliability of Dividend Discount Model in Valuation of Bank Stocks at the Bombay Stock Exchange. International Journal of Research in Commerce and Management, 5(3), 39-45. Recuperado de https://ijrcm.org.in/article info.php?article id=4350

Damodaran, A. (2007). Valuation Approaches and Metrics. A Survey of the Theory and Evidence. Foundations and Trends in Finance, 1(8), 693-784. https://doi.org/10.1561/0500000013

Economatica. (2018). Bases de datos a partir del screening de acciones. Recuperado de https://economatica.com/

Fama, E. F. (1965). Random Walks in Stock Market Prices. Financial Analysts Journal, 21(5), $55-$ 59. https://doi.org/10.2469/faj.v21.n5.55

Fernández, P. (2008). Métodos de Valoración de Empresas. Recuperado de https://pdfs.semanticscholar.org/18d1/5f8f3474991960016b9b5fbf5faafbe4a5d5.pdf

Fondo Monetario Internacional. (2017). Perspectivas de la Economía Mundial. Recuperado de https://www.imf.org/es/Publications/WEO/Issues/2017/07/07/world-economic-outlookupdate-july-2017

Fondo Monetario Internacional. (2018). World Economic Outlook Update. Recuperado de https://www.imf.org/en/Publications/WEO/Issues/2018/07/02/world-economic-outlookupdate-july-2018

Foerster, S. R., \& Sapp, S. G. (2005). The dividend discount model in the long-run: A clinical study. Journal of Applied Finance, 15(2), 55-75. Recuperado de https://ssrn.com/abstract=869545

Göbel, C. (2015). Valoración de Adidas Group (tesis de maestría). Recuperado de http://repositorio.up.edu.pe/handle/11354/1030

Gordon, M., \& Shapiro, E. (1956). Capital Equipment Analysis: The Required Rate of Profit. Management Science, 3(1), 102-110. Recuperado de http://www.jstor.org/stable/2627177

Gordon, M. (1962). The Investment, Financing, and Valuation of the Corporation. Homewood: RD Irwin.

Hernández, B. (2007). Bolsa y estadística bursátil. Madrid: Díaz de Santos. 
Hernández, J. A. (2012). Propuesta metodológica para la construcción de un ranking de emisores en la bolsa de valores de Colombia. Equidad y Desarrollo, (18), 161-209. https://doi.org/10.19052/ed.1795

Investing.com. (2018). Gráfico interactivo, índice MSCI. Recuperado de https://es.investing.com/

Ivanovski, Z., Ivanovska, N., \& Narasanov, Z. (2015). Application of Dividend Discount Model Valuation at Macedonian Stock-Exchange. UTMS Journal of Economics, 6(1), 147-154. Recuperado de http://www.utmsjoe.mk/index.php/past-issues/2-uncategorised/26-vol$\underline{6-n o-1}$

INDEX CALCULATION MSCI. (2018). MSCI dividend points indexes methodology. Recuperado de https://www.msci.com/eqb/methodology/meth docs/MSCl Dividend Points Indexes methodology December 2018.pdf

Jaramillo, F. (2009). Valoración de empresas. Bogotá: Ecoe Ediciones.

Jiang, X., \& Lee, B. (2005). An Empirical Test of the Accounting-Based Residual Income Model and the Traditional Dividend Discount Model. The Journal of Business, 78(4), 1465-1504. https://doi.org/10.1086/430866

Milla, A. (2011). Creación de valor para el accionista. Madrid: Díaz de Santos.

Nasseh, A., \& Strauss, J. (2004). Stock prices and the dividend discount model: did their relation break down in the 1990s? Quarterly Review of Economics and Finance, 44(2), 191-207. https://doi.org/10.1016/j.qref.2003.09.001

Quiroga-Juárez, C. A., \& Villalobos-Escobedo, A. (2015). Análisis del comportamiento bursátil de las principales bolsas financieras en el mundo usando el análisis multivariado (análisis de componentes principales PCA) para el periodo de 2011 a 2014. Revista CEA, 1(2), 25-36. https://doi.org/10.22430/24223182.122

Sim, T., \& H. Wright, R. (2017). Stock valuation using the dividend discount model: An internal rate of return approach. Research in Finance, 33, 19-32. https://doi.org/10.1108/s0196$\underline{382120170000033002}$

Tareq, M. A. (2012). 'Is Residual Income Model (RIM) REALLY Superior to Dividend Discount Model (DDM)?' - A Misconception. IOSR Journal of Business and Management, 5(6), 3644. https://doi.org/10.9790/487X-0563644

Van Horne, J. C. (1997). Administración Financiera (Décima Ed). México: Prentice Hall Hispanoamericana.

Vila, A. (2004). El coste de capital como elemento clave de una valoración. Finanzas \& Contabilidad, 41, 32-36. 